\title{
Editorial
}

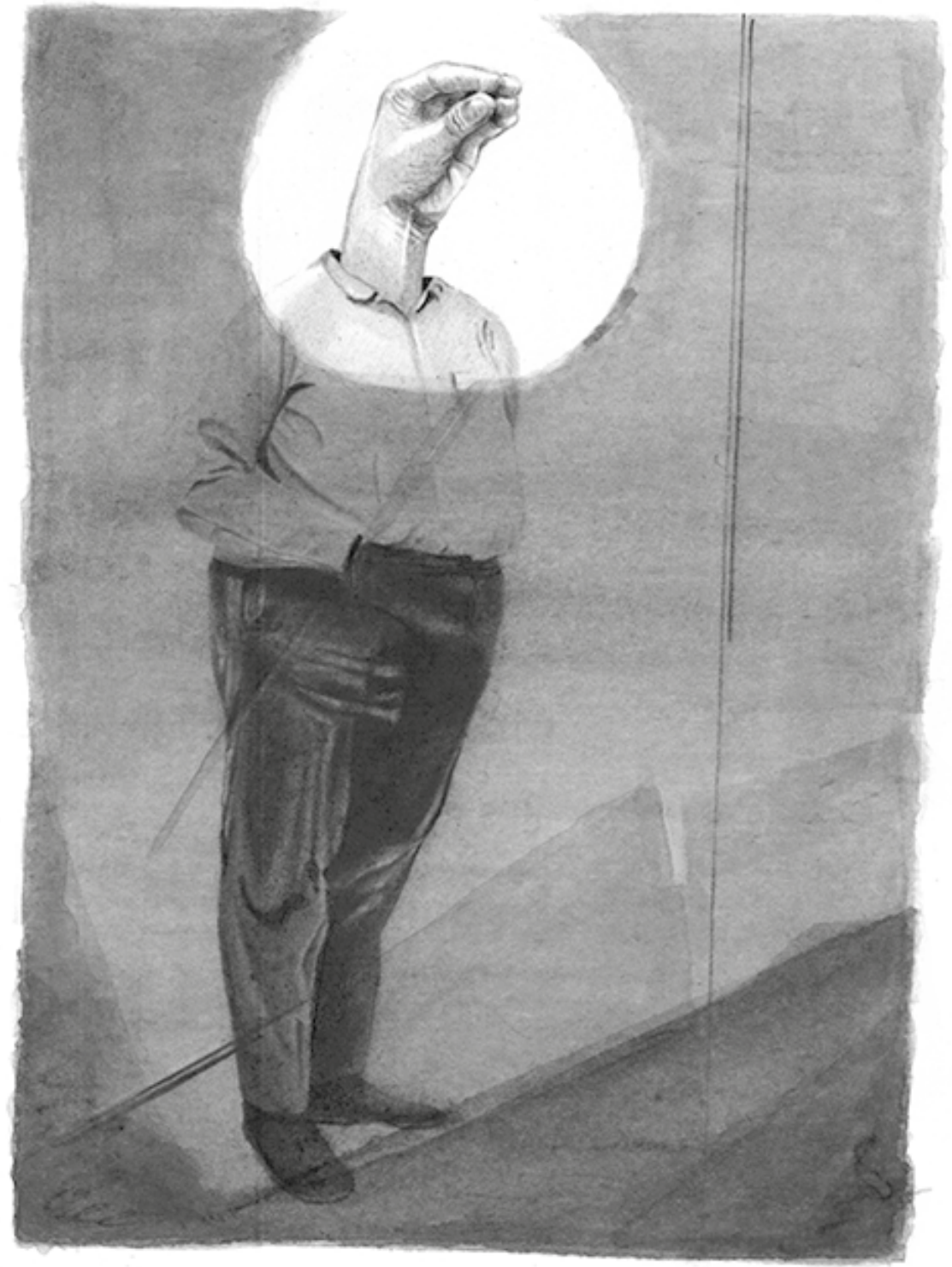

Luis Fernando Salazar Espinosa

Sin título

Lápiz sobre papel

$18 \times 15 \mathrm{~cm}$

2017

Medellín 


\section{Planeación del desarrollo y construcción territorial de la paz}

Durante el primer semestre de 2020, las 1122 entidades administrativas locales en Colombia -que agrupan 1103 municipios, ocho distritos especiales, 18 áreas no municipalizadas y la isla de San Andrés-y sus 32 departamentos tienen la responsabilidad de poner en marcha los procesos de elaboración de sus planes cuatrianuales de desarrollo en un contexto especial de posconflicto, luego de la firma del Acuerdo final para la terminación del conflicto y la construcción de una paz estable y duradera entre el Gobierno nacional y las Fuerzas Armadas Revolucionarias de Colombia-Ejército del Pueblo (FARC-EP) (Gobierno Nacional y FARC-EP, 2016).

La elaboración de los planes territoriales del desarrollo son ejercicios colectivos, realizados en los municipios y departamentos para establecer objetivos y diseñar un conjunto de acciones para construir futuros deseables de desarrollo territorial y transformaciones de las realidades para mejorar el bienestar social. En este sentido, los planes de desarrollo se constituyen en cartas de navegación para que los mandatarios locales y departamentales, y sus respectivos equipos de trabajos - teniendo como restricción las competencias, los recursos y las responsabilidades que le da la ley-, presenten a los ciudadanos un conjunto de estrategias para el mejoramiento de las condiciones de vida de la población, que propicie acciones para una vida diga, que trabaje a partir de una perspectiva de la justicia social, con empleo y seguridad —física, humana, ciudadana y social—, que proteja la naturaleza y los derechos ambientales, y que aborde el desarrollo territorial local —veredal y municipal—, departamental y nacional, e incluso internacional (DNP, 2011; Valencia, 2019).

En esta perspectiva, la planeación del desarrollo territorial adquiere un significado amplio, transversal y estratégico: amplio, porque obliga a pensar lo territorial más allá de la tradicional ordenación jurídico-político de la municipalidad o el departamento, e invita a adoptar una perspectiva de trabajo multinivel, a ver a cada departamento y a sus municipios como parte de una región geográfica; transversal, porque es un ejercicio de la planeación del desarrollo que debe involucrar a todos los sectores y esferas que confluyen 
e intervienen el territorio; y estratégico, porque se debe asumir como un ejercicio de ordenamiento integral y multitemporal de programas, políticas y acciones, entre otros componentes, para que el territorio logre el completo goce de los derechos sociales, colectivos y ambientales.

Para dar cumplimiento a esta labor, los gestores públicos cuentan en Colombia con los lineamientos establecidos en la Ley 152 de 1994, o Ley Orgánica del Plan de Desarrollo, y todos los otros que ofrece el Departamento Nacional de Planeación (DNP). En ellos se indica a los organismos municipales y departamentales tener en cuenta la Constitución Política de 1991 (art. 339), el Plan Nacional de Desarrollo (Ley 1955 de 2019) y los diversos planes, políticas y programas aprobados por los distintos organismos y ámbitos administrativos, como los planes de ordenamiento territorial (POT), las ordenanzas departamentales, los acuerdos municipales, las políticas públicas sectoriales, entre otros.

En especial, la Ley 152 de 1994 establece la necesidad de realizar ejercicios de planeación concertada con las comunidades. Entrega a los gestores públicos el reto de coordinar el trabajo colectivo, para que confluyan en el diseño de los planes de desarrollo municipales y departamentales los actores públicos, sociales y privados. Obliga la ley a que la planeación sea un proceso participativo que involucre a las comunidades de forma directa, tanto en la fase de diagnóstico como en la formulación de los planes de desarrollo. En el proceso de diagnóstico, la ciudadanía debe ayudar a suministrar insumos para realizar balances y evaluar planes anteriores, a señalar vacíos en las políticas previas y en las vigentes, y a identificar nuevas problemáticas. Le dan al diagnóstico el momento propicio para canalizar las demandas ciudadanas y tenerlas en cuenta en la elaboración de los nuevos planes. Y en la fase de diseño, la ciudadanía valida con su presencia las propuestas que se incluyan en el documento, advierten sobre lo deseable y posible, y contribuye a sentir como suya la apuesta estratégica del plan.

Así, la planeación del desarrollo establece como eje la participación ciudadana, comunitaria y política, reconoce la necesidad de la participación de los diversos actores sociales, privados y públicos, y su incidencia en el desarrollo territorial. La norma es enfática en que los planes de desarrollo deben recoger esa diversidad de los intereses y plasmarlos en sus lineamientos de acción. El objetivo es involucrar a la población en el desenvolvimiento de los territorios, que se inserte en las sendas del desarrollo sostenible, el bienestar social y la 
equidad; generar ciudadanos empoderados, comprometidos, participativos e incidentes en las condiciones de vida de los territorios que habitan.

Los gestores públicos tienen una responsabilidad mayor en este primer semestre de 2020, pues además de inyectarle una mirada amplia, transversal y estratégica a la planeación del desarrollo, se tiene el reto de dinamizar los procesos de democracia participativa, movilizando a la ciudadanía en torno a una apuesta colectiva de desarrollo territorial, convertir la formulación de los planes de desarrollo territoriales en un escenario en el que la gente comparta valores, realice apuestas de futuro y trabaje para que se implementen los programas y proyectos (Franco y Mayorga, 2019).

Estos retos se plantean en un contexto de posconflicto. Es claro que los municipios colombianos afrontan por primera vez, luego de la firma del Acuerdo final, el desarrollo territorial en perspectiva de la construcción de la paz. Esta es una tarea constitucionalmente vinculante, en la medida que a través del Acto Legislativo 02 del 2017 se obliga a las instituciones y autoridades del Estado a cumplir de buena fe los puntos establecidos en el Acuerdo del Teatro Colón. Tarea que apenas comienza y que tiene como horizonte de trabajo las siguientes dos décadas.

En este sentido, el mandato constitucional exige que los ejercicios de planeación del desarrollo territorial en Colombia incorporen acciones dirigidas a la implementación del Acuerdo final y a trabajar en la construcción territorial de la paz. Una idea de construcción de paz que, incluso, tiene soporte en los Objetivos de Desarrollo Sostenible de la Organización de las Naciones Unidas (ONU), los cuales ponen como meta «Promover sociedades pacíficas e inclusivas para el desarrollo sostenible, facilitar el acceso a la justicia para todos y crear instituciones eficaces, responsables e inclusivas a todos los niveles» (PNUD, s. f.), y que para su logro el país cuenta, desde hace un par de años, con el Documento Conpes 3918 de 2018.

En Colombia se viene trabajando en este sentido desde hace varios años. Son variados los esfuerzos que desde el segundo gobierno de Juan Manuel Santos (2014-2018) se han ejecutado con la pretensión de avanzar en la implementación del Acuerdo final y en la construcción de la paz con un enfoque territorial. Desde el inicio de las negociaciones con las FARCEP en 2012, los implicados en la Mesa de Conversaciones de La Habana reconocieron la territorialidad de los conflictos, así como la necesidad de 
realizar acciones institucionales y sociales dirigidas a generar condiciones que posibiliten vivir en paz en los territorios: «el conflicto ha afectado más a unos territorios que a otros»y, por tanto, es necesario que se «articulen los esfuerzos y se movilice a la población en esos territorios alrededor de la paz» (Jaramillo, s. f., p. 1).

En concreto, en el punto 1. Reforma Rural Integral del Acuerdo final quedó establecido la necesidad de trabajar en programas de desarrollo con enfoque territorial (PDET), disposición iniciada en 2017 con la selección de 170 municipios con 11000 veredas en 19 departamentos, distribuidos en 16 subregiones (Decreto 893 de mayo de 2017), priorizados por el Departamento Nacional de Planeación (DNP) como los más afectados por el conflicto armado interno y donde se presenta el mayor abandono institucional y la presencia de economías ilícitas manejadas por organizaciones criminales. Estos municipios fueron acompañados durante más de un año por la Agencia de Renovación del Territorio (ART, 2019, febrero 23) para apoyar a las autoridades locales y a las comunidades a redactar los proyectos de inversión y gestión.

Los PDET son instrumentos de gestión pública y de planeación participativa con el que se ha buscado efectuar los programas señalados en el Acuerdo final. Con ellos se viene trabajando en la articulación de los diversos proyectos de inversión social con la paz y conseguir en el mediano y largo plazo cerrar la brecha de desigualdad existente entre el campo y la ciudad. Estos programas tienen para los gestores públicos, encargados de apoyar y elaborar los planes de desarrollo, tanto municipales como departamentales, la doble ventaja de que, primero, deben de servir de insumos para la elaboración de diagnósticos sobre las problemáticas y necesidades de los territorios, pues para su elaboración se realizaron similares actividades a las que se deben ejecutar en la fase de diagnóstico que se plantea para la elaboración de planes de desarrollo; y segundo, que dichos diagnósticos y priorizaciones de proyectos de inversión y gestión se realizaron con la confluencia y alta participación de las comunidades, en una dinámica de planeación participativa de abajo hacia arriba, a partir de núcleos veredales que luego se extienden a los municipios y de allí a las regiones.

Los PDET son una construcción colectiva, fueron las mismas comunidades las encargadas de planificar el desarrollo en su territorio y proponer los proyectos de acuerdo con su contexto; son coherentes con el enfoque territorial, al tener en cuenta las necesidades específicas y las particularidades 
económicas, productivas, sociales, culturales y ambientales; y son un claro ejemplo de implementación de la Ley 152 de 1994, pues se observa una participación de diversos actores, cumpliendo la esencia de ser un proceso de planeación colectiva y no una imposición unilateral del Gobierno, ni una política de desarrollo generalizada para todas las regiones priorizadas.

De esta forma, los gestores públicos y las personas que se ocupen de elaborar los planes de desarrollo municipales y departamentales encontrarán en los PDET una muy buena fuente de información para cumplir su tarea de diagnóstico y diseño. Para los otras 952 administraciones locales y los 13 departamentos no incluidos, la tarea de construir planes coherentes con el mandato constitucional, de cumplir de buena fe los puntos establecidos en el Acuerdo final, se amplía (Documento Conpes 3932 de 2018). Sin embargo, es necesario que todos trabajen para incorporar en los planes todas aquellas normas, acciones, estrategias, programas, políticas, metas, directrices y objetivos para avanzar en la construcción territorial de la paz en Colombia.

Es claro que los municipios y los departamentos dependen para su planificación del desarrollo de los lineamientos que el Estado central determine, pero debe reconocer también que, con buena disposición y sabiendo seleccionar un conjunto de acciones que la misma Constitución Política le señala, pueden contribuir desde sus territorios a la implementación del Acuerdo final y a la construcción de paz. Acciones concretas como, por ejemplo, las relacionadas con la Reforma Rural Integral: a) revisar la política tributaria del municipio o departamento para aumentar tarifas prediales rurales que bien podría ayudar a modificar la alta concentración de la propiedad rural; b) actualización de los catastros rurales municipales y programas de titularización y formalización de tierras rurales; c) promover mayores y mejores niveles de educación en el campo; d) destinar recursos para bienes públicos en el sector rural que permitan cerrar las brechas urbano-rurales; e) establecer políticas públicas agrarias y de conservación ambiental; y f) promover la participación política electoral y de las comunidades alejadas de los cascos urbanos, de tal forma que puedan hacer parte de la toma de decisiones y ejercer mayor control político (Sánchez, 2019, pp. 57-58).

En conclusión, los gestores públicos y los múltiples actores involucrados en la formulación de los planes de desarrollo municipales y departamentales deberán realizar esfuerzos para construir documentos coherentes con el momento histórico en Colombia. Por eso, en este texto se les invita 
a incorporar a la ciudadanía en la construcción de escenarios futuros deseados, deliberados y consensuados colectivamente; a trabajar en la fase de diagnóstico de la formulación de los planes de desarrollo para identificar proyectos dinamizadores de inversión social que permitan construir bases sólidas para la paz y conseguir, en el mediano y largo plazo, cerrar la brecha de desigualdad existente entre el campo y la ciudad; en definitiva, planes que ayuden a dinamizar el desarrollo social, económico y productivo de los territorios y contribuyan a la solución de las causas que dieron origen al largo conflicto armado interno.

Germán Darío Valencia Agudelo (Colombia)*

\section{Referencias bibliográficas}

1. Agencia para la Renovación del Territorio (ART). (2019, febrero 23). Listos los 16 Planes de Acción de los Programas de Desarrollo con Enfoque Territorial -PDET-. Recuperado de https://www.renovacionterritorio.gov.co/Publicaciones/listos_los_16_ planes_de_accin_de_los_programas_de_de_desarrollo_con_enfoque_territorial_ pdet

2. Colombia. Congreso de la República. Ley 152. (15 de julio de 1994). Por la cual se establece la Ley Orgánica del Plan de Desarrollo. Recuperado de https:// colaboracion.dnp.gov.co/CDT/Normatividad/LEY\%20152\%20DE\%201994.pdf

3. Colombia. Congreso de la República. Acto Legislativo 02. (11 de mayo de 2017). Por medio del cual se adiciona un artículo transitorio a la Constitución con el propósito de dar estabilidad y seguridad jurídica al acuerdo final para la terminación del conflicto y la construcción de una Paz Estable y Duradera. Recuperado de https:// www.funcionpublica.gov.co/eva/gestornormativo/norma.php?i $=81573$

4. Colombia. Congreso de la República. Ley 1955. (28 de mayo de 2019). Por el cual se expide el Plan Nacional de Desarrollo 2018-2022. «Pacto por Colombia, pacto por la equidad». Recuperado de https://dapre.presidencia.gov.co/normativa/ normativa/LEY\%201955\%20DEL\%2025\%20DE\%20MAYO\%20DE\%202019.pdf

\footnotetext{
* Director revista Estudios Políticos. Economista. Especialista en Gerencia Social. Magíster en Ciencia Política. Doctor en Estudios Políticos. Grupo Hegemonía, guerras y conflictos, Instituto de Estudios Políticos, Universidad de Antioquia UdeA. Calle 70 No. 52-21, Medellín, Colombia. Correo electrónico: german.valencia@udea.edu.co - Orcid: 0000-0002-6412-6986 - Google Scholar https:// scholar.google.es/citations?user=7Sm8z3MAAAAJ\&hl=es
}

Cómo citar este artículo: Valencia Agudelo, Germán Darío. (2020). Editorial. Planeación del desarrollo y construcción territorial de la paz. Estudios Políticos (Universidad de Antioquia), 57, pp. 10-16. DOI: 10.17533/udea.espo.n57a01 
5. Colombia. Consejo Nacional de Política Económica y Social. Documento Conpes 3918. (15 de marzo de 2018). Estrategia para la implementación de los Objetivos de Desarrollo Sostenible (ODS) en Colombia. Recuperado de https:// colaboracion.dnp.gov.co/CDT/Conpes/Econ\%C3\%B3micos/3918.pdf

6. Colombia. Consejo Nacional de Política Económica y Social. Documento Conpes 3932. (29 de junio de 2018). Lineamientos para la articulación del plan marco de implementación del acuerdo final con los instrumentos de planeación, programación y seguimiento a políticas públicas del orden nacional y territorial. Recuperado de https://colaboracion.dnp.gov.co/CDT/Conpes/Econ\%C3\%B3micos/3932.pdf

7. Colombia. Ministerio de Agricultura y Desarrollo Rural. Decreto Ley 893. (28 de mayo de 2017). Por el cual se crean los Programas de Desarrollo con Enfoque Territorial PDET. Recuperado de http://es.presidencia.gov.co/normativa/normativa/ DECRETO\%20893\%20DEL\% 2028\%20DE\%20MAYO\%20DE\%202017.pdf

8. Departamento Nacional de Planeación (DNP). (2011). Guías para la gestión pública territorial. Planeación para el desarrollo integral en las entidades territoriales. Bogotá, D. C.: DNP.

9. Franco, Omar y Mayorga, Miguel. (2019). La descentralización como agente del cambio: una mirada a través de los Planes de Desarrollo y de Ordenamiento Territorial. Revista Espacios, 40 (16), pp. 1-9. Recuperado de http://www.revistaespacios.com/ a19v40n16/19401607.html

10. Gobierno Nacional y Fuerzas Armadas Revolucionarias de Colombia-Ejército del Pueblo (FARC-EP). (2016). Acuerdo Final para la Terminación del Conflicto y la Construcción de una Paz Estable y Duradera. Bogotá, D. C.: Imprenta Nacional de Colombia.

11. Jaramillo, Sergio. (s. f.). La paz territorial. Interaktive Demokratie. Recuperado de https://interaktive-demokratie.org/files/downloads/La-Paz-Territorial.pdf

12. Programa de las Naciones Unidad para el Desarrollo (PNUD). (s. f.). Objetivo 16: paz, justicia e instituciones sólidas. Recuperado de https://www.undp.org/content/ undp/es/home/sustainable-development-goals/goal-16-peace-justice-and-stronginstitutions.html

13. Sánchez, Cristian. (2019). Concentración de tierra, paz territorial e impuesto predial rural en Antioquia. (Tesis inédita de pregrado). Instituto de Estudios Políticos, Universidad de Antioquia, Medellín.

14. Valencia, Germán. (2019). La paz y la JEP en el Plan Nacional de Desarrollo (2018-2022). Debates, 81, pp. 34-42. 